DOI: 10.1136/annrheumdis-2019-eular.6276

Table 2. Risk factors for cardiovascular mortality (MCV)

\begin{tabular}{lcccc}
\hline \multicolumn{2}{c}{ Bivariate } & \multicolumn{2}{c}{ Multivariate } \\
\hline & HR (IC & $\mathrm{p}$ & HR (IC 95\% & $\mathrm{p}$ \\
& $95 \%)$ & & & \\
\hline Sex & & 0.2 & & 0.1 \\
Arrhythmia & $3.8(1.6-9.2)$ & 0.003 & $3.4(1.4-8.4)$ & 0.007 \\
Number of therapies & $0.6(0.4-0.9)$ & 0.03 & $0.5(0.3-0.9)$ & 0.01 \\
GC withdrawal due to IM & & 0.2 & $0.4(0.1-$ & 0.05 \\
remission & & & $1.005)$ & \\
\hline
\end{tabular}

\section{AB0207 RECEPTOR EXPRESSION OF ANGIOTENSIN TYPE-1 AND 2 ARE DECREASED IN PATIENTS WITH SYSTEMIC SCLEROSIS AND PULMONARY ARTERIAL HYPERTENSION(PAH) AND CORRELATED WITH SEROLOGICAL LEVELS OF NT-PROBNP}

Sebastian Klapa ${ }^{1,2}$, Silke Pitann ${ }^{1}$, Gabriela Marschner ${ }^{1}$, Susanne Riepe ${ }^{1}$, Andreas Koch ${ }^{2}$, Antje Müller ${ }^{1}$, Harald Heidecke ${ }^{3}$, Peter Lamprecht ${ }^{1}$, Gabriela Riemekasten'. 'University of Lübeck, Clinic of Rheumatology and Clinical Immunology, Lübeck, Germany, ${ }^{2}$ Christian Albrechts University Kiel, Institute of experimental Medicine c/o German Naval Medical Institute, Kronshagen, Germany; ${ }^{3}$ CellTrend GmbH, Luckenwalde, Germany

Background: Previous studies identified functional autoantibodies against the angiotensin receptor type-1 (AT1R) and the endothelin receptor type A (ETAR) in about $85 \%$ of the patients with systemic sclerosis (SSc, 1). The antibodies are cross-reactive, agonistic and functionally active by increasing the effects of the natural ligands as well as by specific activation of the receptors $(2,3)$. The levels of the antibodies are associated with clinical findings such as pulmonary arterial hypertension (PAH). Patients with highest antibody levels show worst prognosis and do not respond well to receptor blocker therapy (2-4). Several in vitro effects of the antibodies depend on the antibody levels and on the cell type bearing the receptors. Receptor expression of ETAR and AT1R was highest in early disease (3).

Objectives: To determine predictors of PAH, DU and clinical complications by measuring the antibody levels and the receptor expression on peripheral mononuclear cells in patients with systemic sclerosis.

Methods: The current study analyzed the serological levels of anti-AT1R and anti-ETAR antibodies and the extracellular and intracellular expression of AT1R, AT2R, ETAR and ETBR on circulating CD4pos T cells, CD8pos $T$ cells, CD14pos Macrophages, CD15pos Granulocytes and CD19pos B cells in SSc $(n=41)$ using sandwich ELISA and flow cytometry. Clinical data (PAH, history of digital ulcers, digital-ulcers score, mRSS, pulmonary fibrosis, therapy) and serological markers (ESR, CRP, NT-proBNP) were gathering at the time of serum sampling and every three-month up to 27 month after baseline.

Results: Patients with PAH demonstrated a lower AT1R MFI and AT1R/ AT2R MFI ratio on all PBMC. Levels of NT-proBNP correlated negatively with the AT1R MFI and AT1R/AT2R MFI ratio on all PBMC. The levels of anti-AT1R ab correlated with the NT-proBNP in SSc patients with levels of NT-proBNP $<300 \mathrm{pg} / \mathrm{ml}$. AT1R MFI on T cells, Granulocytes and Macrophages distinguished between pathological and physiological levels of NT-proBNP. Using Log-rank test and Mantel-Cox proportional hazards model, decreased expression of AT1R MFI on T cells, Granulocytes and Macrophages identified trends of deterioration of NT-proBNP over $50 \%$. Conclusion: Expression of AT1R and AT2R on PBMC could be of diagnostic value identifying clinical progress and/or subgroups in SSc. Their role in the pathophysiology, e.g. their impact of endothelial damage, has to be further investigated in SSc.

\section{REFERENCES:}

[1] Riemekasten G. et al., Involvement of functional autoantibodies against vascular receptors in systemic sclerosis. Ann Rheum Dis. 2011 Mar;70 (3):530-6.

[2] Kill A. et al., Autoantibodies to angiotensin and endothelin receptors in systemic sclerosis induce cellular and systemic events associated with disease pathogenesis. Arthritis Res Ther. 2014 Jan 28;16(1):R29.

[3] Becker MO. Et al., Vascular receptor autoantibodies in pulmonary arterial hypertension associated with systemic sclerosis. Am J Respir Crit Care Med. 2014 Oct 1;190(7):808-17.

[4] Günther J. et al., Angiotensin receptor type 1 and endothelin receptor type A on immune cells mediate migration and the expression of $\mathrm{IL}-8$ and CCL18 when stimulated by autoantibodies from systemic sclerosis patients. Arthritis Res Ther. 2014 Mar 11;16(2):R65.
Acknowledgement: We thank Actelion Pharmaceutical $\mathrm{GmbH}$ for their financial support.

Disclosure of Interests: Sebastian Klapa: None declared, Silke Pitann: None declared, Gabriela Marschner: None declared, Susanne Riepe: None declared, Andreas Koch: None declared, Antje Müller: None declared, Harald Heidecke: None declared, Peter Lamprecht: None declared, Gabriela Riemekasten Consultant for: Chugai, F. Hoffmann-La Roche, Speakers bureau: Chugai, F. Hoffmann-La Roche

DOI: 10.1136/annrheumdis-2019-eular.6813

\section{AB0208 UP-REGULATION OF TMEM176A AND TMEM176B IN PERIPHERAL BLOOD CELLS, A POSSIBLE BIOMARKER FOR THE DEVELOPMENT OF EARLY PULMONARY VASCULAR DISEASE IN SYSTEMIC SCLEROSIS}

Yoshinobu Koyama ${ }^{1}$, Soichiro Fuke ${ }^{2}$, Yoshiharu Sato ${ }^{3}$, Akemi Senoh ${ }^{4}$ Toshie Higuchi'. ' 'Japanese Red Cross Okayama Hospital, Rheumatology, Center for Auto Immune Diseases, Okayama, Japan; ${ }^{2}$ Japanese Red Cross Okayama Hospital, Cardiology, Okayama, Japan; ${ }^{3}$ DNA Chip Research Inc, Tokyo, Japan; ${ }^{4}$ Japanese Red Cross Okayama Hospital, Dermatology, Okayama, Japan

Background: Pulmonary arterial hypertension (PAH) is prominent as a vascular involvement of systemic sclerosis (SSc), which remains a leading cause of death in spite of current best treatments. As the pulmonary vascular disease (PVD) can be well compensated for, more than $2 / 3$ of the pulmonary circulation is impaired by the time of diagnosis. However, there were few studies focusing on the molecular mechanisms of PVD at the subclinical stage of PAH in SSc patients.

Objectives: To detect candidate genes involved in the development of PVD before diagnosis of PAH in SSc patients.

Methods: Total of 88 patients who had not met PAH criteria with Raynaud phenomenon $(n=75)$, skin sclerosis $(n=58)$ or SSc-related autoantibody $(n=59)$ was enrolled. To detect the early PVD, exercise Doppler echocardiography (exDE) was carried out. The exercise induced pulmonary arterial hypertension (exPAH) group was segregated from normal response group (exN) with using the reported definition ${ }^{1)}$. The exDE was followed every 6 or 12 months for up to 3 years. For gene expression analysis, total RNAs from whole peripheral blood cells were extracted by PAXgene system and multiplex sequencing was done. To identify candidate genes involved in the pathogenesis of the early PVD, the differentially expressed genes (DEGs) between exPAH and exN group were visualized by volcano plots, a scatter plots calculated to show foldchanges and $p$-values of DEGs. After identifying the candidate genes, weighted gene co-expression network analysis (WGCNA) was performed to find the co-expressed gene cluster. And then, enrichment analysis using gene ontology (GO) terms were performed to investigate roles of the gene cluster in pathogenesis of PVD.

Results: Patients were fall into exPAH group $(n=14)$ and exN group $(n=74)$ at the baseline. After gene expression analysis, 1204 DEGs between exPAH and exN group were identified. Volcano plots indicated that expressions of TMEM176A and TMEM176B were prominent $\left(\log _{2}\right.$ fold-change $>1.25$ and $-\log _{10} \mathrm{p}$-value $>3.5$ ) in exPAH patients. We also found that the expressions of these genes were highly correlated with each other $\left(R^{2}=0.9724, p<0.01\right)$. 11 out of 74 patients $(14.9 \%)$ in exN group progressed to exPAH group during follow-up period $(24.8 \pm 11.3$ months). The expressions of TMEM176A/B at the baseline were significantly up-regulated in the group of clinical progression as compare with others $\left(\log _{2}\right.$ fold-change from the average: TMEM176A: $0.78 \pm 1.31$ vs. $0.35 \pm 1.65 ; p=0.022$, TMEM176B: $0.71 \pm 1.38$ vs. $-0.38 \pm 1.78 ; p=0.034)$. By WGCNA and GO enrichment analysis, the terms, such as related to "apoptosis", "Toll-like receptor signaling" and "dendritic cell differentiation" were found in the co-expressed gene cluster of TMEM176A/B.

Conclusion: Although elucidating the mechanisms for progression of early PVD in SSc remains a major challenge, (1) we found up-regulation of TMEM176A/B was prominent in exPAH group; (2) TMEM176A/B was significantly up-regulated at the baseline in the patients progressed from exN to exPAH group during the follow-up period; (3) the co-expressed gene cluster of TMEM176A/B includes genes related to "apoptosis", "Tolllike receptor signaling" and "dendritic cell differentiation". TMEM176A/B was found initially to be a regulator for DC maturation in mice ${ }^{2)}$ but functions of human ortholog of these genes remain still unclear. However, our findings suggest that the up-regulation of TMEM176A/B in peripheral blood cells is possible to be a biomarker for the development of early PVD in SSc.

\section{REFERENCE:}

[1] R. Naeije, et al., Am J resp and critical care med 187, 576-583 (2013). 2) Condamine, T.et al., J of leuko biol 88, 507-515 (2010). 
Disclosure of Interests: Yoshinobu Koyama Paid instructor for: Asahi Kasei Pharma, Speakers bureau: BMS, Chugai, Ayumi and Eli Lilly, Soichiro Fuke: None declared, Yoshiharu Sato: None declared, Akemi Senoh: None declared, Toshie Higuchi: None declared DOI: 10.1136/annrheumdis-2019-eular.6628

\section{AB0209 A STUDY OF CORRELATION BETWEEN PLATELETS AND LYMPHOCYTE SUBSETS IN SCLEROSIS}

Jie Liang, LI Zhao-Hua, Fangyuan Hu, Ning Yan, LI Xiaofeng, Caihong Wang. the Second Hospital of Shanxi Medical University, Taiyuan, China

Background: Systemic sclerosis (SSc) is an autoimmune disease characterized by vasculopathy, autoimmunity and widespread dermal and visceral fibrosis. There are existing evidence to support excessive platelet activation and their contribution to vascular function and fibrosis. Now we will focus on the immune role of PLT in SSC.

Objectives: By analyzing the correlation between platelet and lymphocyte subsets, CD4 + T cell subsets and disease activity in patients with diffuse SSc (dcSSc)/limitedSSc (IcSSc), and to explore the immune role of PLT in SSC.

Methods: The peripheral blood of 21 stable disease patients, 31 active disease patients and 20 healthy controls $(\mathrm{HC})$ were collected. The clinical data and laboratory indicators of them were enrolled. The T, B, NK lymphocyte subsets and CD4 + T cell subsets were detected by flow cytometry (FCM). The CD4 + T cell subsets contains Th1, Th2, TH17, Treg, Th1/Th2 and Th17/Treg. Non-parametric Kruskal-Wallis $H$ test was performed on multiple independent samples. The correlation between variables was used by Spearman correlation analysis.

Results: The PLT,PCT,MPV,PDW in peripheral blood of the stable group and active group were significantly higher than the $H C \operatorname{group}(P<0.05)$. The amount of $T$ cells in peripheral blood of active group were [1127.80 (796.66,1363.79)], which was lower than the percentage of HC group [1246.44(984.81,1497.84)] and stable group [1428.59(1179.09,2022.88)], there was significant difference between active group and stable group $(Z=6.525, P=0.038)$. The amount of NK cells of the stable group [198.48 (140.22, 302.97)] and active group [143.76(89.00, 230.00)]were significantly lower than the $\mathrm{HC}$ group $[224.81(155.04,350.51)](Z=8.694, P<0.05)$. The amount of Th cells of the stable group[888.11(679.55, 1430.70)] and active group[684.20(385,878)] were higher than the HC group[574.84 $(493.22,728.00)]$, the former was significant, there was significant difference between active group and stable $\operatorname{group}(Z=8.694, \quad P<0.05)$. The Th17/Treg ratio of the stable group $[0.18(0.10,0.34)]$ and active group $[0.21(0.13,028]$ were lower than the HC group $[0.25(0.12,0.31)]$, there was significant difference between active group and $\mathrm{HC}$ group $(Z=0.508$, $\mathrm{P}<0.05)$. The ESR and CRP were positively correlated with PLT, PCT; The amount of Th cells and Th17 cells were positively correlated with PLT,PCT; The amount of Th2 cells and the ratio of CD4+T/CD8+ T were positively correlated with PCT; There was a negative correlation between the ratio of Th1/Th2 and PCT; The amount of Th2 cells was positively correlated with MVP, and the ratio of Th1/Th2 was negatively correlated with MPV.Among these immune cells, NK cells was negatively correlated with ESR and DSS(disease severity scale).

Conclusion: In scleroderma, NK cells are associated with the severity of the disease, and PLT affects the number of Th1/Th2, Th17, and disease activity.

\section{REFERENCES:}

[1] Ntelis K, Solomou E E, Sakkas L, et al. The role of platelets in autoimmunity, vasculopathy and fibrosis. Implications for systemic sclerosis[J]. Seminars in Arthritis and Rheumatism, 2017:S0049017217301105.

[2] Pauling J D, O'Donnell, V. B, Mchugh N J. The contribution of platelets to the pathogenesis of Raynaud"s phenomenon and systemic sclerosis[J]. Platelets, 2013, 24(7):503-515

Acknowledgement: The National Natural Science Fund(81471618); Shanxi Provincial Health Department research project (201201072); Shanxi Provincial Key Research and Development Program (Social Development Field) Project (201803D31119)

Disclosure of Interests: None declared

DOI: 10.1136/annrheumdis-2019-eular.7129
$\mathrm{AB} 0210$

THE GLOBAL EXPRESSION OF MIRNAS AND LNCRNAS IN THE EXOSOMES OF SYSTEMIC SCLEROSIS PLASMA AND NEUTROPHIL AND RELATED FUNCTIONS

LI Liya, Zuo Xiaoxia, DI Liu, Luo Hui, Zhu Honglin. Department of Rheumatology and immunology, Xiangya Hospital, Central South University, Changsha, China

Background: Systemic sclerosis (SSc) is a systemic autoimmune disease with unknown pathogenesis. Exosomes (EXOs) are cell-derived vesicles 30-150 nm in size that contain various mRNAs, microRNAs (miRNAs), long non-coding RNAs (IncRNAs) and proteins. Plasma EXOs play wide roles in various diseased, however, little is known in SSc.

Objectives: Investigate the global expression of miRNAs and IncRNAs in the EXOs of SSc plasma and neutrophil. Explore the potential function of EXOs in the pathogenesis of SSc.

Methods: EXOs were respectively isolated from plasma, cultured neutrophil supernatants, and were identified by transmission electron microscopy. Global expression of miRNAs and IncRNAs form $5 \mathrm{SSc}$ and 5 norma controls was analysis by IlluminaHiSeq 3000 platform. Differentiated expressed and bioinformatics analysis was performed by edgeR, limma package, GO, KEGG, and cytoscape. Furthermore, we used the EXOs stimulated human dermal microvascular endothelial cells (HDMECs) and human primary skin fibroblasts, and explore the potential functions of EXOs.

Results: 1. In plasma EXOs, we identified a total of 37 miRNAs and 479 IncRNAs that showed significant differences between the two groups. Among them, 26 of upregulated miRNAs were involved in the ErbB signalling pathway; 11 of downregulated miRNAs were involved in the MAPK signalling pathway, TGF- $\beta$ signalling pathway, AMPK signalling pathway;299 of upregulated IncRNAs were involved in the PI3K-Akt signalling pathway, p53 signalling pathway;180 of downregulated IncRNAs were involved in lysosome.

2. In neutrophil EXOs, we identified a total of 22 miRNAs and 281 IncRNAs that showed significant differences between the two groups Among them, 12 of upregulated miRNAs were involved in the Wnt signalling pathway; 10 of downregulated miRNAs were involved in the MAPK signalling pathway, AMPK signalling pathway; 119 of upregulated IncRNAs were involved in interleukin-23 signalling pathway;162 of downregulated IncRNAs were involved in the signalling by GPCR, signalling by $\mathrm{NOTCH}$ and TRAIL.

3. After stimulated with SSc plasma EXOs, the expression levels of hasmiR-324-5p and SP1/SMAD2, has-miR-624-5p and PIK3RCB, has-miR 483-5p and MAPK1 were negatively correlated, the expression levels of ENST00000562409.1 and OSMR, ENST00000601511.1 and HMGB1/NRAS were positively correlated, variably in HDMECs and human primary skin fibroblasts.

4. After stimulated with SSc neutrophil EXOs, the expression levels of has-miR-1268a and PRKCA, has-miR-299-3p and IGF1R were negatively correlated, the expression levels of ENST00000520562.1 and IL23R, ENST00000596567.1 and TFDP2, ENST00000608572.1 and HDAC2 were positively correlated, variably in neutrophil, HDMECs and human primary skin fibroblasts.

5. These miRNAs and IncRNAs with consistent expression might be involved in the pathogenesis of SSc EXOs.

Conclusion: Our study identified and confirmed differentially miRNAs and IncRNAs in the neutrophil EXOs and plasma EXOs. Those genes may be involved in the pathological mechanism of SSc, such as has-miR-324 $5 p$ and SP1/SMAD2, has-miR-624-5p and PIK3RCB, has-miR-483-5p and MAPK1, ENST00000520562.1 and IL23R, ENST00000596567.1 and TFDP2, ENST00000608572.1 and HDAC2.

Acknowledgement: This study was funded by grants from the National Key Research and Development Program of China (2016YFC0903900), National Natural Science Foundation of China (81671622), Hunan Provincial Natural Science Foundation (2018JJ3823), Clinical Research Fund of Xiangya Hospital Central South University (2014L10) and Independent Innovation Projects of Central South University (2018zzst290).

Disclosure of Interests: None declared

DOI: 10.1136/annrheumdis-2019-eular.6495 